\title{
Searching Information Mountain on the Java Island to help improve Tourism with the Vector Space Model
}

\author{
Yuri Yuliani ${ }^{1}$, Muhamad Azhar ${ }^{2}$, Ikhwanul Hakim ${ }^{3}$, Sfenrianto $^{4}$, Achmad Rifai ${ }^{5}$ \\ ${ }^{1,2,3}$ Master of Computer Science Postgraduate Program, STMIK Nusa Mandiri , Jakarta, Indonesia, \\ ${ }^{1} 14002311 @$ nusamandiri.ac.id, ${ }^{2}$ muhammadazhar88@gmail.com, ${ }^{3}$ ikhwanulhakim@ymail.com \\ ${ }^{4}$ Information System management Departemen, BINUS Graduate Program, Master of Information System \\ Management, Bina Nusantara University, Jakarta, Indonesia, sfenrianto@binus.edu \\ ${ }^{5}$ Technical Information, STMIK Nusa Mandiri, Jakarta, Indonesia, achmad.acf@nusamandiri.ac.id
}

\begin{abstract}
Mountain expeditions and climbers are not hindered by advances in digital technology at the moment, seen increasing mountain climbing on the island of Java. This is due to the abundance of biodiversity in the mountains of the island of Java such as Mount Gede Pangrango which has many nail trees, at the foot of Mount Rinjani which is surrounded by butterfly species, Mount Merbabu with 70 species of birds, natural savanna with an area of $\pm 300 \mathrm{Ha}$ and has the highest mountain, Mount Semeru with 3,676 meters above sea level. The potential and natural wealth can attract tourists to travel there so that it can be a factor to develop the economy in the area. This research helps tourism to search information mountain in Java Island by using the Vector Space Model (VSM) algorithm. The algorithm using to data preparation, tokenizing, filtering, stemming, calculation, and finally document ranking framework. Generated 14 sample data from search results on Google with the query "mountain on the island of Java". VSM algorithm runs well enough to produce the highest ranking of 3.716189934 .
\end{abstract}

Key words : Searching Information, Mountain Java, Tourism, VSM.

\section{INTRODUCTION}

The passion among nature lovers to be able to see the beauty on the top of the mountain does not disappear with technological advancements in the current digital era. Seen from the expedition and mountaineering that is increasingly carried out by the climbers in the mountains of Indonesia, especially mountain climbing on the island of Java [1].

Java Island is one of the islands in Indonesia which has many volcanoes, high mountains and has high biodiversity mountains such as Semeru Mountain, (3,676 mdpl) [2], Gunung Gede Pangrango which has many nail trees [3], then at the foot of Mount Rinjani which is abundant with butterfly species [4], Merbabu mountain with 70 species of birds [5], baluran mountain which has a natural savanna, namely bekol savanna with an area of $\pm 300 \mathrm{Ha}$ which is the largest savanna on the island of Java [6].

With the advantages possessed by the mountains on the island of Java can make many natural connoisseurs who hunt for the beauty that is owned by every mountain on this island of Java. Because the potential or superior natural wealth in an area can be a factor in regional economic development [7].

This study is intended to determine the extent to which tourists know the attractions on the largest island in Indonesia. By using the Vector Space Model (VSM) is expected to help tourism on the island of Java in promoting tourist attractions, by helping to improve the information [8] to increase the number of tours, especially climbers.

\section{RESEARCH FRAMEWORK}

\subsection{Information Retrival}

Formation retrieval is a science to get information in the form of text collected from documents based on queries. The information presented is a list of documents based on queries that have been assessed based on similarities using the similarity function [9]. The ranking results obtained from documents that have relevance to the query, for the level of relevance is a subjective thing that is influenced by factor topics, time, sources of information and user objectives [10].

\subsection{VSM Model}

Vector Space Model introduces tf-IDF weighting as the term weight scheme. Each weight that has a frequency is called a (tf) factor that measures the frequency of occurrences in a document or text and the frequency document factor (IDF) measures the inverse of the number of documents containing a query or document [11]. With a framework like the following: 


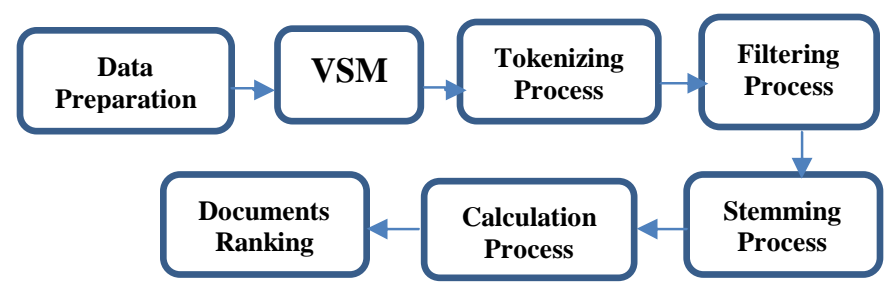

Figure 1: Research Framework

The data taken is secondary data taken on a mountain search on the island of Java on Google. Then the data will be processed using the Vector Space Model (SVM) to calculate the number of values obtained based on keywords by the process of tokenizing, filtering, stemming, calculation and ranking.

Then proceed with the tokenizing process. Tokenizing itself is a process of breaking down word by word in a sentence or document, to get weight on each word [12], which is calculated using Term Frequency (Tf) and Document Frequency (Df). After the tokenizing process, it is followed by a filtering process which functions to solve words and discard words that are not important for classification [13].

The next stage is the process of the stem which is the process of producing basic words by removing affixes to a word [14], numbers, and who have punctuation. Then do the calculation process and end with ranking on the document

\section{DESCUSSION}

\subsection{Research Data Preparation}

Research data obtained from the results of a search on google with the query "gunung di pulau jawa". Then take 14 sample titles from search results on Google, including:

[D.1] Terpikat Pesona Gunung Ciremai, Gunung Tertinggi di Jawa Barat.

[D.2] Menyaksikan Sunrice Gunung Semeru, Cantik Bikin Rindu Mendaki

[D.3] Semeru dan Empat Gunung Mempesona di Malang

[D.4] Menggapai Puncak Semeru, Atap Pulau Jawa

[D.5] Gunung Slamet, Gunung Tertinggi Kedua di Pulau Jawa dengan Tinggi $3428 \mathrm{Mdpl}$

[D.6] Menyaksikan Sunrise Gunung di Jawa, Cantik Bikin Rindu Mendaki

[D.7] Gunung Agrapura, Trek Pendakian Terpanjang di Jawa

[D.8] Legenda Gunung Semeru Sebagai Paku Bumi Pulau Jawa

[D.9] Gunung Slamet Pusat Spiritualitas di Jawa

[D.10] Keindahan Gunung Bromo, Semeru, Prau dan gede-Pangrango di Pulau Jawa ini Bikin Susah Move-on

[D.11] Tertutup Awan, Gunung Sumbing Disebut

Nampak Mengerikan

[D.12] Letusan Gunung Merapi yang Konon Mengubah Sejarah Jawa

[D.13] Gunung Api Nglanggeran, Kebumen, Kaldera pasir Tengger merupakan gunung api Purba di Pulau Jawa

[D.14] Gunung Papandayan merupakan gunung yang ada sabana di jawa

\subsection{Tokenizing Process}

After the data is collected, a tokenizing process is carried out, with the following results:

Table 1: Tokenizing Process

\begin{tabular}{|c|c|c|c|}
\hline terpikat & Pesona & gunung & Ceremai \\
\hline gunung & Tertinggi & di & Jawa \\
\hline Barat & $\begin{array}{l}\text { menyaksika } \\
\mathrm{n}\end{array}$ & sunrise & Gunung \\
\hline Semeru & Cantik & bikin & Rindu \\
\hline Pendaki & Semeru & dan & Empat \\
\hline Gunung & mempesona & di & Malang \\
\hline Menggapai & Puncak & semeru & Atap \\
\hline Pulau & Jawa & gunung & Slamet \\
\hline Gunung & tertinggi & kedua & Di \\
\hline Pulau & Jawa & dengan & Tinggi \\
\hline 3428 & Mdpl & $\begin{array}{l}\text { menyaksika } \\
\mathrm{n}\end{array}$ & Sunrise \\
\hline Gunung & $\mathrm{Di}$ & jawa & Cantik \\
\hline Bikin & Rindu & mendaki & Gunung \\
\hline Argapura & Trek & pendakian & Terpanjang \\
\hline Di & Jawa & legenda & Gunung \\
\hline Semeru & Sebagai & paku & Bumi \\
\hline Pulau & Jawa & gunung & Slamet \\
\hline Pusat & spiritualitas & di & Jawa \\
\hline Keindahan & Gunung & bromo & Semeru \\
\hline Prau & Dan & gede & Pangrango \\
\hline Di & Pulau & jawa & Ini \\
\hline Bikin & Susah & move & On \\
\hline Tertutup & Awan & gunung & Sumbing \\
\hline Disebut & Nampak & mengerikan & Letusan \\
\hline Gunung & Merapi & yang & Konon \\
\hline Mengubah & Sejarah & jawa & Gunung \\
\hline Api & nglanggeran & kebumen & kalder \\
\hline Pasir & Tengger & merupakan & Gunung \\
\hline Api & Purba & di & Pulau \\
\hline Jawa & Gunung & papandayan & Merupakan \\
\hline Gunung & Yang & ada & Sabana \\
\hline Di & Jawa & & \\
\hline
\end{tabular}


Yuri Yuliani et al., International Journal of Emerging Trends in Engineering Research, 8(2), February 2020, 317 - 322

\subsection{Filtering Process}

After getting the words that have been processed at the tokenizing stage, then the filtering stage is then carried out with the results of the data. Table 2 shown filtering process in the study.

Table 2: Filtering Process

\begin{tabular}{|l|l|l|l|}
\hline ada & api & argapura & atap \\
\hline awan & barat & bikin & bromo \\
\hline bumi & cantik & ceremai & dan \\
\hline dengan & di & disebut & empat \\
\hline gede & gunung & ini & jawa \\
\hline kalder & kebumen & kedua & keindahan \\
\hline konon & legenda & letusan & malang \\
\hline mdpl & mempesona & mendaki & mengerikan \\
\hline menggapai & mengubah & menyaksikan & menyaksikan \\
\hline merapi & merupakan & move & nampak \\
\hline $\begin{array}{l}\text { nglanggera } \\
\text { n }\end{array}$ & on & paku & pangrango \\
\hline papandayan & pasir & pendaki & pendakian \\
\hline pesona & prau & pulau & puncak \\
\hline purba & pusat & rindu & sabana \\
\hline sebagai & sejarah & semeru & slamet \\
\hline spiritualitas & sumbing & sunrise & susah \\
\hline tengger & terpanjang & terpikat & tertinggi \\
\hline tertutup & Tinggi & trek & Yang \\
\hline 3.4 Stemming Process & & \\
\hline
\end{tabular}

\subsection{Stemming Process}

From the data generated at the filtering stage of 72 words, it will proceed to the stemming stage which produces 61 words as follows:

Table 3: Stemming Precess

\begin{tabular}{|c|c|c|c|}
\hline ada & api & argapura & atap \\
\hline awan & bagai & barat & bikin \\
\hline bromo & bumi & cantik & ceremai \\
\hline daki & dua & empat & gapai \\
\hline gede & gunung & indah & jawa \\
\hline kalder & kebumen & konon & legenda \\
\hline letus & malang & mdpl & merapi \\
\hline nampak & negeri & nglanggeran & paku \\
\hline pangrango & panjang & papandayan & pasir \\
\hline pendaki & pesona & pikat & prau \\
\hline pulau & puncak & purba & pusat \\
\hline rindu & rupa & sabana & saksi \\
\hline sebut & sejarah & semeru & slamet \\
\hline spiritualitas & sumbing & sunrise & susah \\
\hline tengger & tinggi & trek & tutup \\
\hline ubah & & & \\
\hline
\end{tabular}

\subsection{Calculation Process}

Look for the number of words in a document (tf) and documents containing the searched word (df) (see figure 2)

\begin{tabular}{|c|c|c|c|c|c|c|c|c|c|c|c|c|c|c|c|}
\hline \multirow{2}{*}{ Token } & \multicolumn{15}{|c|}{ tf } \\
\hline & $\mathrm{Q}$ & D1 & D2 & D3 & D4 & \begin{tabular}{l|l}
4 D5 \\
\end{tabular} & D6 & D7 & D8 & D9 & D10 & \begin{tabular}{|l|} 
D11 \\
\end{tabular} & D12 & D13 & D14 \\
\hline ada & 0 & 0 & 0 & 0 & 0 & 0 & 0 & 0 & 0 & 0 & 0 & 0 & 0 & 0 & 1 \\
\hline api & 0 & 0 & 0 & 0 & 0 & 0 & 0 & 0 & 0 & 0 & 0 & 0 & 0 & 1 & 0 \\
\hline argapura & 0 & 0 & 0 & 0 & 0 & 0 & 0 & 1 & 0 & 0 & 0 & 0 & 0 & 0 & 0 \\
\hline atap & 0 & 0 & 0 & 0 & 1 & 0 & 0 & 0 & 0 & 0 & 0 & 0 & 0 & 0 & 0 \\
\hline awan & 0 & 0 & 0 & 0 & 0 & 0 & 0 & 0 & 0 & 0 & 0 & 1 & 0 & 0 & 0 \\
\hline bagai & 0 & 0 & 0 & 0 & 0 & 0 & 0 & 0 & 1 & 0 & 0 & 0 & 0 & 0 & 0 \\
\hline barat & 0 & 1 & 0 & 0 & 0 & 0 & 0 & 0 & 0 & 0 & 0 & 0 & 0 & 0 & 0 \\
\hline bikin & 0 & 0 & 1 & 0 & 0 & 0 & 1 & 0 & 0 & 0 & 1 & 0 & 0 & 0 & 0 \\
\hline bromo & 0 & 0 & 0 & 0 & 0 & 0 & 0 & 0 & 0 & 0 & 1 & 0 & 0 & 0 & 0 \\
\hline bumi & 0 & 0 & 0 & 0 & 0 & 0 & 0 & 0 & 1 & 0 & 0 & 0 & 0 & 0 & 0 \\
\hline cantik & 0 & 0 & 1 & 0 & 0 & 0 & 1 & 0 & 0 & 0 & 0 & 0 & 0 & 0 & 0 \\
\hline ceremai & 0 & 1 & 0 & 0 & 0 & 0 & 0 & 0 & 0 & 0 & 0 & 0 & 0 & 0 & 0 \\
\hline daki & 0 & 0 & 0 & 0 & 0 & 0 & 1 & 1 & 0 & 0 & 0 & 0 & 0 & 0 & 0 \\
\hline dua & 0 & 0 & 0 & 0 & 0 & 1 & 0 & 0 & 0 & 0 & 0 & 0 & 0 & 0 & 0 \\
\hline empat & 0 & 0 & 0 & 1 & 0 & 0 & 0 & 0 & 0 & 0 & 0 & 0 & 0 & 0 & 0 \\
\hline gapai & 0 & 0 & 0 & 0 & 1 & 0 & 0 & 0 & 0 & 0 & 0 & 0 & 0 & 0 & 0 \\
\hline gede & 0 & 0 & 0 & 0 & 0 & 0 & 0 & 0 & 0 & 0 & 1 & 0 & 0 & 0 & 0 \\
\hline gunung & 1 & 1 & 1 & 1 & 0 & 1 & 1 & 1 & 1 & 1 & 1 & 1 & 1 & 1 & 1 \\
\hline indah & 0 & 0 & 0 & 0 & 0 & 0 & 0 & 0 & 0 & 0 & 1 & 0 & 0 & 0 & 0 \\
\hline jawa & 1 & 1 & 0 & 0 & 1 & 1 & 1 & 1 & 1 & 1 & 1 & 1 & 1 & 1 & 1 \\
\hline kalder & 0 & 0 & 0 & 0 & 0 & 0 & 0 & 0 & 0 & 0 & 0 & 0 & 0 & 1 & 0 \\
\hline kebumen & 0 & 0 & 0 & 0 & 0 & 0 & 0 & 0 & 0 & 0 & 0 & 0 & 0 & 1 & 0 \\
\hline konon & 0 & 0 & 0 & 0 & 0 & 0 & 0 & 0 & 0 & 0 & 0 & 0 & 1 & 0 & 0 \\
\hline legenda & 0 & 0 & 0 & 0 & 0 & 0 & 0 & 0 & 1 & 0 & 0 & 0 & 0 & 0 & 0 \\
\hline letus & 0 & 0 & 0 & 0 & 0 & 0 & 0 & 0 & 0 & 0 & 0 & 0 & 1 & 0 & 0 \\
\hline malang & 0 & 0 & 0 & 1 & 0 & 0 & 0 & 0 & 0 & 0 & 0 & 0 & 0 & 0 & 0 \\
\hline mdp1 & 0 & 0 & 0 & 0 & 0 & 1 & 0 & 0 & 0 & 0 & 0 & 0 & 0 & 0 & 0 \\
\hline merapi & 0 & 0 & 0 & 0 & 0 & 0 & 0 & 0 & 0 & 0 & 0 & 0 & 1 & 0 & 0 \\
\hline nampak & 0 & 0 & 0 & 0 & 0 & 0 & 0 & 0 & 0 & 0 & 0 & 1 & 0 & 0 & 0 \\
\hline ngeri & 0 & 0 & 0 & 0 & 0 & 0 & 0 & 0 & 0 & 0 & 0 & 1 & 0 & 0 & 0 \\
\hline nglanggeran & 0 & 0 & 0 & 0 & 0 & 0 & 0 & 0 & 0 & 0 & 0 & 0 & 0 & 1 & 0 \\
\hline paku & \begin{tabular}{|l|} 
\\
\end{tabular} & 0 & 0 & 0 & 0 & 0 & 0 & 0 & 1 & 0 & 0 & 0 & 0 & 0 & 0 \\
\hline pangrango & 0 & 0 & 0 & 0 & 0 & 0 & 0 & 0 & 0 & 0 & 1 & 0 & 0 & 0 & 0 \\
\hline panjang & 0 & 0 & 0 & 0 & 0 & 0 & 0 & 1 & 0 & 0 & 0 & 0 & 0 & 0 & 0 \\
\hline papandayan & 0 & 0 & 0 & 0 & 0 & 0 & 0 & 0 & 0 & 0 & 0 & 0 & 0 & 0 & 1 \\
\hline pasir & 0 & 0 & 0 & 0 & 0 & 0 & 0 & 0 & 0 & 0 & 0 & 0 & 0 & 1 & 0 \\
\hline pendaki & 0 & 0 & 1 & 0 & 0 & 0 & 0 & 0 & 0 & 0 & 0 & 0 & 0 & 0 & 0 \\
\hline pesona & 0 & 1 & 0 & 1 & 0 & 0 & 0 & 0 & 0 & 0 & 0 & 0 & 0 & 0 & 0 \\
\hline pikat & 0 & 1 & 0 & 0 & 0 & 0 & 0 & 0 & 0 & 0 & 0 & 0 & 0 & 0 & 0 \\
\hline prav & 0 & 0 & 0 & 0 & 0 & 0 & 0 & 0 & 0 & 0 & 1 & 0 & 0 & 0 & 0 \\
\hline pulav & 1 & 0 & 0 & 0 & 1 & 1 & 0 & 0 & 1 & 0 & 1 & 0 & 0 & 1 & 0 \\
\hline puncak & 0 & 0 & 0 & 0 & 1 & 0 & 0 & 0 & 0 & 0 & 0 & 0 & 0 & 0 & 0 \\
\hline purba & 0 & 0 & 0 & 0 & 0 & 0 & 0 & 0 & 0 & 0 & 0 & 0 & 0 & 1 & 0 \\
\hline pusat & 0 & 0 & 0 & 0 & 0 & 0 & 0 & 0 & 0 & 1 & 0 & 0 & 0 & 0 & 0 \\
\hline rindu & 0 & 0 & 1 & 0 & 0 & 0 & 1 & 0 & 0 & 0 & 0 & 0 & 0 & 0 & 0 \\
\hline rupa & 0 & 0 & 0 & 0 & 0 & 0 & 0 & 0 & 0 & 0 & 0 & 0 & 0 & 1 & 1 \\
\hline sabana & 0 & 0 & 0 & 0 & 0 & 0 & 0 & 0 & 0 & 0 & 0 & 0 & 0 & 0 & 1 \\
\hline sakssi & 0 & 0 & 1 & 0 & 0 & 0 & 1 & 0 & 0 & 0 & 0 & 0 & 0 & 0 & 0 \\
\hline sebut & 0 & 0 & 0 & 0 & 0 & 0 & 0 & 0 & 0 & 0 & 0 & 1 & 0 & 0 & 0 \\
\hline sejarah & 0 & 0 & 0 & 0 & 0 & 0 & 0 & 0 & 0 & 0 & 0 & 0 & 1 & 0 & 0 \\
\hline semerv & 0 & 0 & 1 & 1 & 1 & 0 & 0 & 0 & 1 & 0 & 1 & 0 & 0 & 0 & 0 \\
\hline slamet & 0 & 0 & 0 & 0 & 0 & 1 & 0 & 0 & 0 & 1 & 0 & 0 & 0 & 0 & 0 \\
\hline spiritualitz & 0 & 0 & 0 & 0 & 0 & 0 & 0 & 0 & 0 & 1 & 0 & 0 & 0 & 0 & 0 \\
\hline sumbing & 0 & 0 & 0 & 0 & 0 & 0 & 0 & 0 & 0 & 0 & 0 & 1 & 0 & 0 & 0 \\
\hline sunrise & 0 & 0 & 1 & 0 & 0 & 0 & 1 & 0 & 0 & 0 & 0 & 0 & 0 & 0 & 0 \\
\hline susah & 0 & 0 & 0 & 0 & 0 & 0 & 0 & 0 & 0 & 0 & 1 & 0 & 0 & 0 & 0 \\
\hline tengger & 0 & 0 & 0 & 0 & 0 & 0 & 0 & 0 & 0 & 0 & 0 & 0 & 0 & 1 & 0 \\
\hline tinggi & 0 & 1 & 0 & 0 & 0 & 1 & 0 & 0 & 0 & 0 & 0 & 0 & 0 & 0 & 0 \\
\hline trek & 0 & 0 & 0 & 0 & 0 & 0 & 0 & 1 & 0 & 0 & 0 & 0 & 0 & 0 & 0 \\
\hline tutup & 0 & 0 & 0 & 0 & 0 & 0 & 0 & 0 & 0 & 0 & 0 & 1 & 0 & 0 & 0 \\
\hline bah & 0 & 0 & 0 & 0 & 0 & 0 & 0 & 0 & 0 & 0 & 0 & 0 & 1 & 0 & 0 \\
\hline
\end{tabular}

Figure 2: Grading Per-Word Value 
Yuri Yuliani et al., International Journal of Emerging Trends in Engineering Research, 8(2), February 2020, 317 - 322

A. Calculation Phase of Inverse Document Frequency (IDF)

After knowing the value of tf and df, then search for Inverse Document Frequency (IDF) using the equation IDFi $=$ LOG . D/df. Where, D : document total; df: Many documents contain the words that are searched for IDF calculation results for "gunung" data are Know : $\mathrm{D}=14$ ([D.1], [D.2], [D.3], [D.5], [D.6], [D.7], [D.8], [D.9], [D.10], [D.11], [D.12], [D.13], [D.14]). Df (gunung) $=13$. Thus, IDF $=$ LOG (14/ $13)=\operatorname{LOG} 4=0,0322$.

\section{B. Calculating Document Weight (W)}

Calculate the weight of a document by using an equation: $\mathrm{Wf}_{\mathrm{t}, \mathrm{d})}=\mathrm{tf}_{\mathrm{t}, \mathrm{d}} * \mathrm{IDF}_{\mathrm{t} .}$. The results of calculating the weight of documents for the "gunung" data in document 1 (one) [D.1] are $\mathrm{tf}_{(\mathrm{td}) \text { gunung }}=1 * \mathrm{IDF}_{\mathrm{t}(\mathrm{gunun} g)}=0,0322 \mathrm{Dit}: \mathrm{Wf}_{(\mathrm{td}) \text { gunung }} \mathrm{m}=$ $\mathrm{tf}_{(\mathrm{td}) \text { gunung }} * \mathrm{IDF}_{\mathrm{t}(\text { gunung) }}=1 * 0,0322=0,0322$.

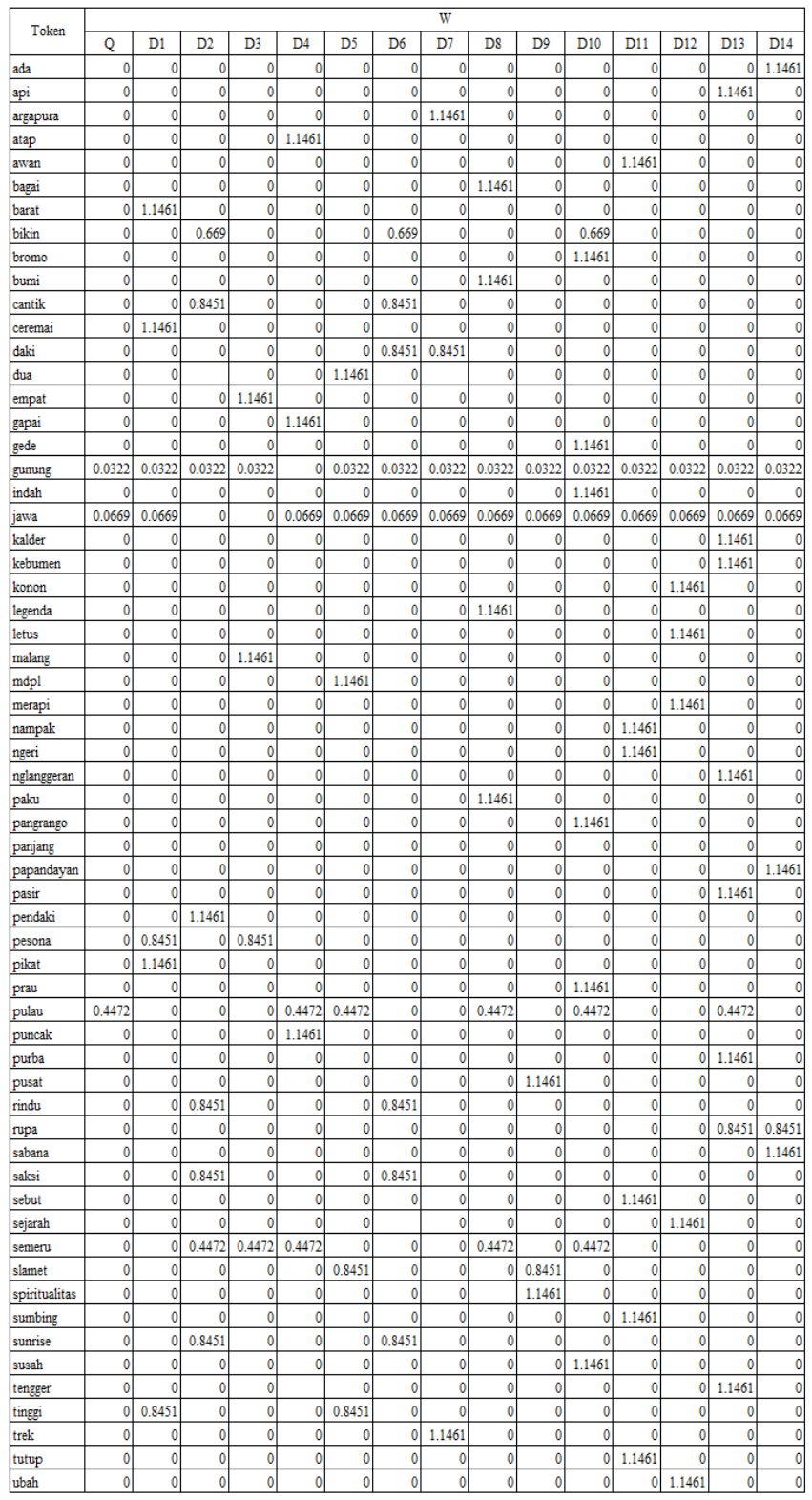

Figure 3: Calculation of Word Weightl
C. Calculation of Distance Q-D

Calculate distances from $\mathrm{Q}$ to $\mathrm{D}$ using the equation: $\operatorname{Sqrt}(\mathrm{Q}) \operatorname{Sqrt}\left(\sum_{j=1}^{\mathrm{n}} \mathrm{Q}_{j}^{2}\right)$. The results of the calculation of the distance calculation for the data "mountain" in document 1 (one) [D.1] is $(0.0322) *(0.0322)=0.0011$

\begin{tabular}{|c|c|c|c|c|c|c|c|c|c|c|c|c|c|c|}
\hline \multicolumn{15}{|c|}{ Jarak Q-D } \\
\hline$Q^{\prime 2}$ & D1^2 & $\mathrm{D} 2 / 2$ & $\mathrm{D} 3^{\wedge} 2$ & $\mathrm{D} 4{ }^{\wedge} 2$ & $\mathrm{D} 5^{\wedge} 2$ & $\mathrm{D} 6{ }^{\wedge} 2$ & D7/2 & D8^2: & $|\mathrm{D} 9 \cdot 2| \mathrm{I}$ & D10.2 & $\mathrm{D} 11^{\wedge} 2$ & $2 \mathrm{D} 12^{\wedge} 2$ & D13^2 & $214^{\wedge} 2$ \\
\hline 0 & 0 & 0 & 0 & 0 & 0 & 0 & 0 & 0 & 0 & 0 & 0 & 0 & 0 & 1.314 \\
\hline 0 & 0 & 0 & 0 & 0 & 0 & 0 & 0 & 0 & 0 & 0 & 0 & 0 & 1.314 & 0 \\
\hline 0 & 0 & 0 & 0 & 0 & 0 & 0 & \begin{tabular}{|l|}
1.314 \\
\end{tabular} & 0 & 0 & 0 & 0 & 0 & 0 & 0 \\
\hline 0 & 0 & 0 & 0 & 1.314 & 0 & 0 & 0 & 0 & 0 & 0 & 0 & 0 & 0 & 0 \\
\hline 0 & 0 & 0 & 0 & 0 & 0 & 0 & 0 & 0 & 0 & 0 & 1.314 & 0 & 0 & 0 \\
\hline 0 & 0 & 0 & 0 & 0 & 0 & 0 & 0 & 1.314 & 0 & 0 & 0 & 0 & 0 & 0 \\
\hline 0 & 1.314 & 0 & 0 & 0 & 0 & 0 & 0 & 0 & 0 & 0 & 0 & 0 & 0 & 0 \\
\hline 0 & 0 & 0.448 & 0 & 0 & 0 & 0.448 & 0 & 0 & 0 & \begin{tabular}{|l|}
0.448 \\
\end{tabular} & 0 & 0 & 0 & 0 \\
\hline 0 & 0 & 0 & 0 & 0 & 0 & 0 & 0 & 0 & 0 & $\mid 1.314$ & 0 & 0 & 0 & 0 \\
\hline 0 & 0 & 0 & 0 & 0 & 0 & 0 & 0 & 1.314 & 0 & 0 & 0 & 0 & 0 & 0 \\
\hline 0 & 0 & 0.714 & 0 & 0 & 0 & 0.714 & 0 & 0 & 0 & 0 & 0 & 0 & 0 & 0 \\
\hline 0 & 1.314 & 0 & 0 & 0 & 0 & 0 & 0 & 0 & 0 & 0 & 0 & 0 & 0 & 0 \\
\hline 0 & 0 & 0 & 0 & 0 & 0 & 0.714 & \begin{tabular}{|l|}
0.714 \\
\end{tabular} & 0 & 0 & 0 & 0 & 0 & 0 & 0 \\
\hline 0 & 0 & 0 & 0 & 0 & 1.314 & 0 & 0 & 0 & 0 & 0 & 0 & 0 & 0 & 0 \\
\hline 0 & 0 & 0 & 1.314 & 0 & 0 & 0 & 0 & 0 & 0 & 0 & 0 & 0 & 0 & 0 \\
\hline 0 & 0 & 0 & 0 & 1.314 & 0 & 0 & 0 & 0 & 0 & 0 & 0 & 0 & 0 & 0 \\
\hline 0 & 0 & 0 & 0 & 0 & 0 & 0 & 0 & 0 & 0 & 1.314 & 0 & 0 & 0 & 0 \\
\hline 0.00104 & 0.001 & \begin{tabular}{|l|}
0.001 \\
\end{tabular} & \begin{tabular}{|l|}
0.001 \\
\end{tabular} & 0 & 0.001 & 0.001 & \begin{tabular}{|l|}
0.001 \\
\end{tabular} & \begin{tabular}{|l|}
0.001 \\
\end{tabular} & \begin{tabular}{|l|}
0.001 \\
\end{tabular} & \begin{tabular}{|l|}
0.001 \\
\end{tabular} & 0.001 & 0.001 & \begin{tabular}{|l|}
0.001 \\
\end{tabular} & 0.001 \\
\hline 0 & 0 & 0 & 0 & 0 & 0 & 0 & 0 & 0 & 0 & 1.314 & 0 & 0 & 0 & 0 \\
\hline 0.00448 & 0.004 & 0 & 0 & 0.004 & 0.004 & 0.004 & 0.004 & 0.004 & 0.004 & 0.004 & 0.004 & 0.004 & 0.004 & 0.004 \\
\hline 0 & 0 & 0 & 0 & 0 & 0 & 0 & 0 & 0 & 0 & 0 & 0 & 0 & \begin{tabular}{|l|}
1.314 \\
\end{tabular} & 0 \\
\hline 0 & 0 & 0 & 0 & 0 & 0 & 0 & 0 & 0 & 0 & 0 & 0 & 0 & \begin{tabular}{|l|}
1.314 \\
\end{tabular} & 0 \\
\hline 0 & 0 & 0 & 0 & 0 & 0 & 0 & 0 & 0 & 0 & 0 & 0 & 1.314 & 0 & 0 \\
\hline 0 & 0 & 0 & 0 & 0 & 0 & 0 & 0 & \begin{tabular}{|l|}
1.314 \\
\end{tabular} & 0 & 0 & 0 & 0 & 0 & 0 \\
\hline 0 & 0 & 0 & 0 & 0 & 0 & 0 & 0 & 0 & 0 & 0 & 0 & 1.314 & 0 & 0 \\
\hline 0 & 0 & 0 & \begin{tabular}{|l|}
1.314 \\
\end{tabular} & 0 & 0 & 0 & 0 & 0 & 0 & 0 & 0 & 0 & 0 & 0 \\
\hline 0 & 0 & 0 & 0 & 0 & 1.314 & 0 & 0 & 0 & 0 & 0 & 0 & 0 & 0 & 0 \\
\hline 0 & 0 & 0 & 0 & 0 & 0 & 0 & 0 & 0 & 0 & 0 & 0 & 1.314 & 0 & 0 \\
\hline 0 & 0 & 0 & 0 & 0 & 0 & 0 & 0 & 0 & 0 & 0 & 1.314 & 0 & 0 & 0 \\
\hline 0 & 0 & 0 & 0 & 0 & 0 & 0 & 0 & 0 & 0 & 0 & \begin{tabular}{|l|}
1.314 \\
\end{tabular} & 0 & 0 & 0 \\
\hline 0 & 0 & 0 & 0 & 0 & 0 & 0 & 0 & 0 & 0 & 0 & 0 & 0 & 1.314 & 0 \\
\hline 0 & 0 & 0 & 0 & 0 & 0 & 0 & 0 & \begin{tabular}{|l|}
1.314 \\
\end{tabular} & 0 & 0 & 0 & 0 & 0 & 0 \\
\hline 0 & 0 & 0 & 0 & 0 & 0 & 0 & 0 & 0 & 0 & \begin{tabular}{|l|}
1.314 \\
\end{tabular} & 0 & 0 & 0 & 0 \\
\hline 0 & 0 & 0 & 0 & 0 & 0 & 0 & \begin{tabular}{|l|l|}
1.314 \\
\end{tabular} & 0 & 0 & 0 & 0 & 0 & 0 & 0 \\
\hline 0 & 0 & 0 & 0 & 0 & 0 & 0 & 0 & 0 & 0 & 0 & 0 & 0 & 0 & \begin{tabular}{|l|}
1.314 \\
\end{tabular} \\
\hline 0 & 0 & 0 & 0 & 0 & 0 & 0 & 0 & 0 & 0 & 0 & 0 & 0 & $\mid 1.314$ & 0 \\
\hline 0 & 0 & 1.314 & 0 & 0 & 0 & 0 & 0 & 0 & 0 & 0 & 0 & 0 & 0 & 0 \\
\hline 0 & \begin{tabular}{|l|}
0.714 \\
\end{tabular} & 0 & \begin{tabular}{|l|}
0.714 \\
\end{tabular} & 0 & 0 & 0 & 0 & 0 & 0 & 0 & 0 & 0 & 0 & 0 \\
\hline 0 & 1.314 & 0 & 0 & 0 & 0 & 0 & 0 & 0 & 0 & 0 & 0 & 0 & 0 & 0 \\
\hline 0 & 0 & 0 & 0 & 0 & 0 & 0 & 0 & 0 & 0 & \begin{tabular}{|l|}
1.314 \\
\end{tabular} & 0 & 0 & 0 & 0 \\
\hline 0.19995 & 0 & 0 & 0 & 0.2 & 0.2 & 0 & 0 & 0.2 & 0 & 0.2 & 0 & 0 & 0.2 & 0 \\
\hline 0 & 0 & 0 & 0 & 1.314 & 0 & 0 & 0 & 0 & 0 & 0 & 0 & 0 & 0 & 0 \\
\hline 0 & 0 & 0 & 0 & 0 & 0 & 0 & 0 & 0 & 0 & 0 & 0 & 0 & 1.314 & 0 \\
\hline 0 & 0 & 0 & 0 & 0 & 0 & 0 & 0 & 0 & \begin{tabular}{|l|}
1.314 \\
\end{tabular} & 0 & 0 & 0 & 0 & 0 \\
\hline 0 & 0 & 0.714 & 0 & 0 & 0 & 0.714 & 0 & 0 & 0 & 0 & 0 & 0 & 0 & 0 \\
\hline 0 & 0 & 0 & 0 & 0 & 0 & 0 & 0 & 0 & 0 & 0 & 0 & 0 & 0.714 & 0.714 \\
\hline 0 & 0 & 0 & 0 & 0 & 0 & 0 & 0 & 0 & 0 & 0 & 0 & 0 & 0 & 1.314 \\
\hline 0 & 0 & \begin{tabular}{|l|}
0.714 \\
\end{tabular} & 0 & 0 & 0 & 0.714 & 0 & 0 & 0 & 0 & 0 & 0 & 0 & 0 \\
\hline 0 & 0 & 0 & 0 & 0 & 0 & 0 & 0 & 0 & 0 & 0 & 1.314 & 0 & 0 & 0 \\
\hline 0 & 0 & 0 & 0 & 0 & 0 & 0 & 0 & 0 & 0 & 0 & 0 & 1.314 & 0 & 0 \\
\hline 0 & 0 & 0.2 & 0.2 & 0.2 & 0 & 0 & 0 & 0.2 & 0 & 0.2 & 0 & 0 & 0 & 0 \\
\hline 0 & 0 & 0 & 0 & 0 & 0.714 & 0 & 0 & 0 & \begin{tabular}{|l|}
0.714 \\
\end{tabular} & 0 & 0 & 0 & 0 & 0 \\
\hline 0 & 0 & 0 & 0 & 0 & 0 & 0 & 0 & 0 & \begin{tabular}{|l|}
1.314 \\
\end{tabular} & 0 & 0 & 0 & 0 & 0 \\
\hline 0 & 0 & 0 & 0 & 0 & 0 & 0 & 0 & 0 & 0 & 0 & \begin{tabular}{|l|}
1.314 \\
\end{tabular} & 0 & 0 & 0 \\
\hline 0 & 0 & \begin{tabular}{|l|}
0.714 \\
\end{tabular} & 0 & 0 & 0 & \begin{tabular}{|l|}
0.714 \\
\end{tabular} & 0 & 0 & 0 & 0 & 0 & 0 & 0 & 0 \\
\hline 0 & 0 & 0 & 0 & 0 & 0 & 0 & 0 & 0 & 0 & \begin{tabular}{|l|}
1.314 \\
\end{tabular} & 0 & 0 & 0 & 0 \\
\hline 0 & 0 & 0 & 0 & 0 & 0 & 0 & 0 & 0 & 0 & 0 & 0 & 0 & 1.314 & 0 \\
\hline 0 & \begin{tabular}{|l|}
0.714 \\
\end{tabular} & 0 & 0 & 0 & \begin{tabular}{|l|}
0.714 \\
\end{tabular} & 0 & 0 & 0 & 0 & 0 & 0 & 0 & 0 & 0 \\
\hline 0 & 0 & 0 & 0 & 0 & 0 & 0 & 1.314 & 0 & 0 & 0 & 0 & 0 & 0 & 0 \\
\hline 0 & 0 & 0 & 0 & 0 & 0 & 0 & 0 & 0 & 0 & 0 & \begin{tabular}{|l|}
1.314 \\
\end{tabular} & 0 & 0 & 0 \\
\hline 0 & 0 & 0 & 0 & 0 & 0 & 0 & 0 & 0 & 0 & 0 & 0 & 1.314 & 0 & 0 \\
\hline
\end{tabular}

Figure 4: Calculation of Distance Q - D

Sqrt $(\mathrm{Q})=\operatorname{Sqrt}(0,205468031)=0,453285817$.

$\operatorname{Sqrt}\left(\sum_{\mathrm{j}=1}^{\mathrm{n}} \mathrm{Q}_{\mathrm{j}}^{2}\right) \quad[\mathrm{D} .1]=\operatorname{Sqrt}(5,374727543)=2,31834586$

$\operatorname{Sqrt}\left(\sum_{\mathrm{j}=1}^{\mathrm{n}} \mathrm{Q}_{\mathrm{j}}{ }_{\mathrm{j}}\right) \quad[\mathrm{D} .2]=2,195205798$

$\operatorname{Sqrt}\left(\sum_{\mathrm{j}=1}^{\mathrm{n}} \mathrm{Q}_{\mathrm{j}}{ }_{\mathrm{j}}\right) \quad[\mathrm{D} .3]=1,882125342$

$\operatorname{Sqrt}\left(\sum_{\mathrm{j}=1}^{\mathrm{n}} \mathrm{Q}_{\mathrm{j}}{ }_{\mathrm{j}}\right) \quad[\mathrm{D} .4]=2,084516948$

Sqrt $\left(\sum_{\mathrm{j}=1}^{\mathrm{n}} \mathrm{Q}^{2}{ }_{\mathrm{j}}\right) \quad[\mathrm{D} .5]=2,064235542$

$\operatorname{Sqrt}\left(\sum_{\mathrm{j}=1}^{\mathrm{n}} \mathrm{Q}_{\mathrm{j}}{ }_{\mathrm{j}}\right) \quad$ [D.6] $=2,006001317$

$\operatorname{Sqrt}\left(\sum_{\mathrm{j}=1}^{\mathrm{n}} \mathrm{Q}_{\mathrm{j}}{ }_{\mathrm{j}}\right)$ [D.7] $=2,158827656$

$\operatorname{Sqrt}\left(\sum_{\mathrm{j}=1}^{\mathrm{n}} \mathrm{Q}_{\mathrm{j}}^{2}\right) \quad[\mathrm{D} .8]=2,379045236$ 
Yuri Yuliani et al., International Journal of Emerging Trends in Engineering Research, 8(2), February 2020, 317 - 322

$\operatorname{Sqrt}\left(\sum_{\mathrm{j}=1}^{\mathrm{n}} \mathrm{Q}_{\mathrm{j}}^{2}\right)$ [D.9] $=1,829460951$

$\operatorname{Sqrt}\left(\sum_{\mathrm{j}=1}^{\mathrm{n}} \mathrm{Q}_{\mathrm{j}}{ }_{\mathrm{j}}\right) \quad[\mathrm{D} .10]=2,955443326$

$\operatorname{Sqrt}\left(\sum_{\mathrm{j}=1}^{\mathrm{n}} \mathrm{Q}_{\mathrm{j}}{ }_{\mathrm{j}}\right) \quad[\mathrm{D} .11]=2,808411396$

$\operatorname{Sqrt}\left(\sum_{\mathrm{j}=1}^{\mathrm{n}} \mathrm{Q}_{\mathrm{j}}^{2}\right) \quad[\mathrm{D} .12]=2,563896468$

D. Calculation of DOT

The DOT product calculation uses the equation:

$\operatorname{Sum}\left(\mathrm{Q} * \mathrm{D}_{\mathrm{i}}\right)=\sum_{\mathrm{j}=1}^{\mathrm{n}} \mathrm{Q}_{\mathrm{j}} \mathrm{D}_{\mathrm{i}, \mathrm{j}}$

(4)

\begin{tabular}{|c|c|c|c|c|c|c|c|c|c|c|c|c|c|c|c|}
\hline \multicolumn{16}{|c|}{ Menghitung DOT } \\
\hline$Q^{*} \mathrm{D} 1$ & $\mathrm{Q}^{*} \mathrm{D} 2$ & Q*D3 & $Q^{*} \mathrm{D} 4$ & $Q^{*} \mathrm{D} 5$ & $Q^{*} \mathrm{D} 6$ & $Q^{*} \mathrm{D} 7$ & $Q^{*} D 8$ & & $\mathrm{Q} * \mathrm{D} 9$ & $Q^{*} \mathrm{D} 1 \mathrm{~d}$ & $\mathrm{Q}^{*} \mathrm{D} 11$ & & 2*D12 & $Q^{*} \mathrm{D} 13$ & $\mathrm{Q} * \mathrm{D} 14$ \\
\hline 0 & 0 & 0 & 0 & 0 & 0 & 0 & & 0 & 0 & 0 & 0 & & 0 & 0 & 0 \\
\hline 0 & 0 & 0 & 0 & 0 & 0 & 0 & & 0 & 0 & 0 & 0 & 0 & 0 & 0 & 0 \\
\hline 0 & 0 & 0 & 0 & 0 & 0 & 0 & & 0 & 0 & 0 & 0 & 0 & 0 & 0 & 0 \\
\hline 0 & 0 & 0 & 0 & 0 & 0 & 0 & & 0 & 0 & 0 & 0 & 0 & 0 & 0 & 0 \\
\hline 0 & 0 & 0 & 0 & 0 & 0 & 0 & & 0 & 0 & 0 & 0 & & 0 & 0 & 0 \\
\hline 0 & 0 & 0 & 0 & 0 & 0 & 0 & & 0 & 0 & 0 & 0 & 0 & 0 & 0 & 0 \\
\hline 0 & 0 & 0 & 0 & 0 & 0 & 0 & & 0 & 0 & 0 & 0 & 0 & 0 & 0 & 0 \\
\hline 0 & 0 & 0 & 0 & 0 & 0 & 0 & & 0 & 0 & 0 & 0 & 0 & 0 & 0 & 0 \\
\hline 0 & 0 & 0 & 0 & 0 & 0 & 0 & & 0 & 0 & 0 & 0 & 0 & 0 & 0 & 0 \\
\hline 0 & 0 & 0 & 0 & 0 & 0 & 0 & 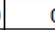 & 0 & 0 & 0 & 0 & 0 & 0 & 0 & 0 \\
\hline 0 & 0 & 0 & 0 & 0 & 0 & 0 & 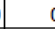 & 0 & 0 & 0 & 0 & 0 & 0 & 0 & 0 \\
\hline 0 & 0 & 0 & 0 & 0 & 0 & 0 & & 0 & 0 & 0 & 0 & & 0 & 0 & 0 \\
\hline 0 & 0 & 0 & 0 & 0 & 0 & 0 & 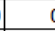 & 0 & 0 & 0 & 0 & & 0 & 0 & 0 \\
\hline 0 & 0 & 0 & 0 & 0 & 0 & 0 & & 0 & 0 & 0 & 0 & 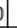 & 0 & 0 & 0 \\
\hline 0 & 0 & 0 & 0 & 0 & 0 & 0 & & 0 & 0 & 0 & 0 & 0 & 0 & 0 & 0 \\
\hline 0 & 0 & 0 & 0 & 0 & 0 & 0 & c & 0 & 0 & 0 & 0 & 0 & 0 & 0 & 0 \\
\hline 0 & 0 & 0 & 0 & 0 & 0 & 0 & & 0 & 0 & 0 & 0 & 0 & 0 & 0 & 0 \\
\hline 1.073 & \begin{tabular}{|l|}
1.073 \\
\end{tabular} & 1.073 & 0 & \begin{tabular}{|l|}
1.073 \\
\end{tabular} & \begin{tabular}{|l|}
1.073 \\
\end{tabular} & 1.073 & 1.07 & & 1.073 & \begin{tabular}{|l|}
1.073 \\
\end{tabular} & 1.073 & & 1.073 & 1.073 & \begin{tabular}{|l|} 
\\
\end{tabular} \\
\hline 0 & 0 & 0 & 0 & 0 & 0 & 0 & c & 0 & 0 & 0 & 0 & & 0 & 0 & 0 \\
\hline 2.009 & 0 & 0 & 2.009 & 2.009 & 2.009 & 2.009 & 2.009 & & 2.009 & 2.009 & 2.009 & & 2.009 & 2.009 & 2.009 \\
\hline 0 & 0 & 0 & 0 & 0 & 0 & 0 & 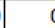 & 0 & 0 & 0 & 0 & & 0 & 0 & 0 \\
\hline 0 & 0 & 0 & 0 & 0 & 0 & 0 & & 0 & 0 & 0 & 0 & 0 & 0 & 0 & 0 \\
\hline 0 & 0 & 0 & 0 & 0 & 0 & 0 & & 0 & 0 & 0 & 0 & 0 & 0 & 0 & 0 \\
\hline 0 & 0 & 0 & 0 & 0 & 0 & 0 & 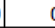 & 0 & 0 & 0 & 0 & D & 0 & 0 & 0 \\
\hline 0 & 0 & 0 & 0 & 0 & 0 & 0 & & 0 & 0 & 0 & 0 & 0 & 0 & 0 & 0 \\
\hline 0 & 0 & 0 & 0 & 0 & 0 & 0 & & 0 & 0 & 0 & 0 & 0 & 0 & 0 & 0 \\
\hline 0 & 0 & 0 & 0 & 0 & 0 & 0 & 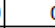 & 0 & 0 & 0 & 0 & D & 0 & 0 & 0 \\
\hline 0 & 0 & 0 & 0 & 0 & 0 & 0 & & 0 & 0 & 0 & 0 & 0 & 0 & 0 & 0 \\
\hline 0 & 0 & 0 & 0 & 0 & 0 & 0 & & 0 & 0 & 0 & 0 & 0 & 0 & 0 & 0 \\
\hline 0 & 0 & 0 & 0 & 0 & 0 & 0 & 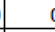 & 0 & 0 & 0 & 0 & f & 0 & 0 & 0 \\
\hline 0 & 0 & 0 & 0 & 0 & 0 & 0 & & 0 & 0 & 0 & 0 & 0 & 0 & 0 & 0 \\
\hline 0 & 0 & 0 & 0 & 0 & 0 & 0 & 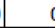 & 0 & 0 & 0 & 0 & D & 0 & 0 & 0 \\
\hline 0 & 0 & 0 & 0 & 0 & 0 & 0 & c & 0 & 0 & 0 & 0 & f & 0 & 0 & 0 \\
\hline 0 & 0 & 0 & 0 & 0 & 0 & 0 & & 0 & 0 & 0 & 0 & & 0 & 0 & 0 \\
\hline 0 & 0 & 0 & 0 & 0 & 0 & 0 & 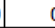 & 0 & 0 & 0 & 0 & 0 & 0 & 0 & 0 \\
\hline 0 & 0 & 0 & 0 & 0 & 0 & 0 & c & 0 & 0 & 0 & 0 & D & 0 & 0 & 0 \\
\hline 0 & 0 & 0 & 0 & 0 & 0 & 0 & & 0 & 0 & 0 & 0 & 0 & 0 & 0 & 0 \\
\hline 0 & 0 & 0 & 0 & 0 & 0 & 0 & c & 0 & 0 & 0 & 0 & & 0 & 0 & 0 \\
\hline 0 & 0 & 0 & 0 & 0 & 0 & 0 & & 0 & 0 & 0 & 0 & D & 0 & 0 & 0 \\
\hline 0 & 0 & 0 & 0 & 0 & 0 & 0 & & 0 & 0 & 0 & 0 & 0 & 0 & 0 & 0 \\
\hline 0 & 0 & 0 & 0.04 & 0.04 & 0 & 0 & 0.04 & & 0 & 0.04 & 0 & & 0 & 0.04 & 0 \\
\hline 0 & 0 & 0 & 0 & 0 & 0 & 0 & c & 0 & 0 & 0 & 0 & 0 & 0 & 0 & 0 \\
\hline 0 & 0 & 0 & 0 & 0 & 0 & 0 & & 0 & 0 & 0 & 0 & 0 & 0 & 0 & 0 \\
\hline 0 & 0 & 0 & 0 & 0 & 0 & 0 & c & 0 & 0 & 0 & 0 & & 0 & 0 & 0 \\
\hline 0 & 0 & 0 & 0 & 0 & 0 & 0 & & 0 & 0 & 0 & 0 & 0 & 0 & 0 & 0 \\
\hline 0 & 0 & 0 & 0 & 0 & 0 & 0 & & 0 & 0 & 0 & 0 & 0 & 0 & 0 & 0 \\
\hline 0 & 0 & 0 & 0 & 0 & 0 & 0 & c & 0 & 0 & 0 & 0 & & 0 & 0 & 0 \\
\hline 0 & 0 & 0 & 0 & 0 & 0 & 0 & & 0 & 0 & 0 & 0 & & 0 & 0 & 0 \\
\hline 0 & 0 & 0 & 0 & 0 & 0 & 0 & & 0 & 0 & 0 & 0 & & 0 & 0 & 0 \\
\hline 0 & 0 & 0 & 0 & 0 & 0 & 0 & 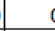 & 0 & 0 & 0 & 0 & & 0 & 0 & 0 \\
\hline 0 & 0 & 0 & 0 & 0 & 0 & 0 & & 0 & 0 & 0 & 0 & & 0 & 0 & 0 \\
\hline 0 & 0 & 0 & 0 & 0 & 0 & 0 & & 0 & 0 & 0 & 0 & & 0 & 0 & 0 \\
\hline 0 & 0 & 0 & 0 & 0 & 0 & 0 & 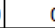 & 0 & 0 & 0 & 0 & & 0 & 0 & 0 \\
\hline 0 & 0 & 0 & 0 & 0 & 0 & 0 & & 0 & 0 & 0 & 0 & & 0 & 0 & 0 \\
\hline 0 & 0 & 0 & 0 & 0 & 0 & 0 & & 0 & 0 & 0 & 0 & & 0 & 0 & 0 \\
\hline 0 & 0 & 0 & 0 & 0 & 0 & 0 & c & 0 & 0 & 0 & 0 & & 0 & 0 & 0 \\
\hline 0 & 0 & 0 & 0 & 0 & 0 & 0 & & 0 & 0 & 0 & 0 & & 0 & 0 & 0 \\
\hline 0 & 0 & 0 & 0 & 0 & 0 & 0 & & 0 & 0 & 0 & 0 & & 0 & 0 & 0 \\
\hline 0 & 0 & 0 & 0 & 0 & 0 & 0 & c & 0 & 0 & 0 & 0 & & 0 & 0 & 0 \\
\hline 0 & 0 & 0 & 0 & 0 & 0 & 0 & & 0 & 0 & 0 & 0 & & 0 & 0 & 0 \\
\hline 0 & 0 & 0 & 0 & 0 & 0 & 0 & 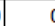 & 0 & 0 & 0 & 0 & 0 & 0 & 0 & 0 \\
\hline
\end{tabular}

Figure 5: Calculation of DOT Product
Results:

[D.1] $=3,0817$

[D.2] $=1,073$

[D.3] $=1,073$

[D. 4$]=2,0487$

[D.5] $=3,1217$

[D.6] $=3,0817$

[D.7] $=3,0817$

[D. 8$]=3,1217$

[D.9] $=3,0817$

[D.10] $=3,1217$

[D.11] $=3,0817$

[D.12] $=3,0817$

[D.13] $=3,1217$

[D.14] $=3,0817$

\section{E. Calculating Similarity}

Calculate the Cosine value of the angle between the vector queries of each document using the formula:

Consine $\theta D i=\frac{Q * D}{|Q| *\|D i\|}$

Cosinus [D.1] $=((0.453285817) *(2,318345864)) /(3,0817)$

Cosinus [D.2] $=((0.453285817) *(2,195205798)) /(1,073)$

Cosinus [D.3] $=((0.453285817) *(1,882125342)) /(1,073)$

Cosinus [D.4] $=((0.453285817) *(2,084516948)) /(2,0487)$

Cosinus $[\mathrm{D} .5]=((0.453285817) *(2,064235542)) /(3,1217)$

Cosinus [D.6] $=((0.453285817) *(2,006001317)) /(3,0817)$

Cosinus [D.7] $=((0.453285817) *(2,158827656)) /(3,0817)$

Cosinus [D.8] $=((0.453285817) *(2,379045236)) /(3,1217)$

Cosinus [D.9] $=((0.453285817) *(1,829460951)) /(3,0817)$

Cosinus [D.10] $=((0.453285817) *(2,955443326)) /(3,1217)$

Cosinus [D.11] $=((0.453285817) *(2,808411396)) /(3,0817)$

Cosinus [D.12] $=((0.453285817) *(1,648809)) /(3,0817)$

Cosinus [D.13] $=((0.453285817) *(2,563896468)) /(3,1217)$

Cosinus [D.14] $=((0.453285817) *(2,158827656)) /(3,0817)$

\section{DOCUMENTS RANGKING}

From the results of the Vector Space Model analysis we get a ranking of 14 calculated documents, namely:

Ranking 1 : [D.9] with value 3,716189934

Ranking 2 : [D.6] with value 3,389142526

Ranking 3 : [D.5] with value 3,336280562

Ranking 4 : [D.7] and [D.14] with value 3,149220529

Ranking 5 : [D.1] with value 2,93252289

Ranking 6 : [D.8] with value 2,894803685

Ranking 7 : [D.12] with value 2,651676641

Ranking 8 : [D.11] with value 2,420807856

Ranking 9 : [D.10] with value 2,330232103

Ranking 10 : [D.4] with value 2,16822849

Ranking 11 : [D.1] with value 2,165411716

Ranking 12 : [D.3] with value 1,257705759

Ranking 13 : [D.2] with value 1,078331646 


\section{CONCLUSION}

The algorithm used in this study shows good results with 14 document sample data. The ranking process uses the stages of the VSM (Vector Space Model) algorithm with the highest value of 3.716189934 which is owned by document 9 from the ranking which produces 13 ranks. The results of the algorithm used in the case of mountain data search on the island of Java can be proven to run well.

This research development still needs to be done which consists of data samples and can be developed using other methods that can get better results in this case.

\section{REFERENCES}

1. Yuliyana, I. A., Februariyanti, H., \& Santoso, D. B. Weather Underground Untuk Layanan Cuaca Pada Sistem Pendakian Gunung. Prosiding Sintak, 83, 2018.

2. Wulandari, A., \& Rahmawati, R. D. Tingkat Ploidi Paku Sayur (Diplazium Esculentum) Pada Ketinggian Yang Berbeda Di Gunung Semeru. Edubiotik, 59, 2018. https://doi.org/10.33503/ebio.v3i02.98

3. Maulidia, A., Sedayu, A., Sakti, D. P., Puspita, E. D., Kusumaningtiyas, F., Ristanto, R. H., Et Al. Keanekaragaman Tanaman Paku (Pteridophyta) Di Jalur Ciwalen Taman Nasional Gunung Gede Pangrango, Jawa Barat. Biosfer, J.Bio. \& Pend.Bio., 29, 2018.

4. Sumiati, Idrus, A. A., \& Ilhamdi, L. Keanekaragaman Kupu-Kupu (Subordo Rhopalocera) Di Kawasan Hutan Jeruk Manis. Prosiding Seminar Nasional Pendidikan Biologi, 399-400, 2018.

5. Aditya, Nugroho, G. D., Jauhar, M. F., \& Sunarto. Keanekaragaman Burung Diurnal Dan Potensi Burung Sebagai Objek Daya Tarik Avitourism Di Taman Nasional Gunung Merbabu, Jawa Tengah. PROS SEM NAS MASY BIODIV INDON, 362-263. 2019

6. Nugroho, H. T. Pengembangan Taman Nasional Baluran Sebagai Destinasiwisata Unggulan Berbasis Alam Di Situbondo Jawa Timur. Jurnal Ilmiah Domestic Case Study. 2019

7. Destiningsih, R., Acha, A., \& Septiani, Y. (2019). Analisis Potensi Wilayah Provinsi Jawa Tengah (Studi Kasus : Tahun 2010-2016). Jurnal REP (Riset Ekonomi Pembangunan), 74, 2019. https://doi.org/10.31002/rep.v4i1.1343

8. Lakshmi, D., Rani, K. P., \& Reddy, M. P. (2019). A Comparative Study of Navigation Techniques and Information. International Journal of Advanced Trends in Computer Science and Engineering, 10.

9. Prasetyo, V. R., Hartanto, B., \& Mulyono, A. A. Penentuan Pembimbing Tugas Akhir Mahasiswa Jurusan Teknik Informatika Universitas Surabaya Dengan Metode Dice Coefficient. TEKNIKA, 45, 2019.

10. Fauziah, S., Sulistyowati, D. N., \& Asra, T. Optimasi Algoritma Vector Space Model Denganalgoritma K-Nearest Neighbour Padapencarian Judul Artikel Jurnal. Jurnal Pilar Nusa Mandiri, 22, 2019
11. Heristian, S., Kautsar, H. A., \& Sayfulloh, A. Rancang Bangun Information Retrieval System (IRS) Kamus Bahasa-Sunda.Com Dengan Metode Vector Space Model (VSM). Jurnal Ilmu Pengetahuan Dan Teknologi Komputer, 66, 2019.

12. Mahendrajaya, R., Buntoro, G. A., \& Setyawan, M. B. (2019). Analisis Sentimen Pengguna Gopay Menggunakan Metode Lexicon Based Dan Support Vector Machine. Komputek , 58, 2019. https://doi.org/10.24269/jkt.v3i2.270

13. Afandi, M. I., Adiwijaya, \& Astuti, W. Klasifikasi Multilabel Pada Hadis Bukhari Terjemahan Bahasa Indonesia Menggunakan Mutual Information Dan Support Vector Machine. E-Proceeding Of Engineering, 9103, 2019

14. Subagyo, I., Yulianto, L. D., Permadi, W., Dewantara, A. W., \& Hartanto, A. D. Sentiment Analisis Review Film Di IMDB Menggunakan Algoritma SVM. Jurnal Sistem Informasi Dan Teknologi Informasi, 48. 2019 